\title{
The importance of surveillance in the control of influenza
}

\author{
Claude Hannoun, PhD
}

\begin{abstract}
C HANNoun. The importance of surveillance in the control of influenza. Can J Infect Dis 1993;4(5):263266. Surveillance of influenza has important practical uses for controlling the spread and the severity of epidemic episodes. The participation of sentinel practitioners directly involved in primary patient care is essential and can be promoted by active surveillance networks working in real time with a strong coordinating structure. The Groupes régionaux d'observation de la Grippe (GROG) system, elaborated in France since 1984 on these principles, has allowed a short and middle term prevision of outbreaks and the isolation of a large number of influenza strains.
\end{abstract}

Key Words: Epidemiological indicators, Influenza surveillance, Sentinel networks, Vaccine strategy

\section{L'importance de la surveillance épidémiologique pour la lutte contre la grippe}

RÉSUMÉ: La surveillance de la grippe a d'importantes applications pratiques dans la lutte contre cette maladie pour en limiter l'extension et la gravité. La participation de médecins praticiens situés en première ligne, au contact direct de la population de tous âges et de toutes conditions est un élément essentiel de cette surveillance et doit être favorisée par l'eutremise de réseaux de surveillance épidémiologique. Ces réseaux de surveillance active doivent fonctionner en temps réel et être très rigoureusement coordonnés. Les réseaux Group régionaux d'observation de la Grippe (GROG), mis en place en France depuis 1984 ont démontré leur aptitude à donner des informations précises et précoces sur l'apparition et le déroulement des épidémies de grippe et ont permis d'isoler un grand nombre de souches d'influenza.

$\mathrm{T}$ HE HISTORY OF INFLUENZA SHOWS THAT EPIDEMICS, AND especially pandemics extending across the globe, can be devastating. In addition, milder but more frequent influenza episodes cause thousands of deaths as well as considerable economic losses annually. These episodes generally start in rural central China and spread to the rest of the world within a few months. Many theories have been proposed to explain this peculiar phenomenon and there is evidence implicating swine and avian influenza viruses in the development of complex gene rearrangements in the human influenza viruses which confer upon some strains a specific degree of increased virulence. Influenza viruses are, however, independently of this phenomenon, highly variable and escape immune mechanisms through progressive and continuous variations of their external components, through processes known as antigenic 'shift' and 'drift'.

Whatever the cause of influenza pandemics, we have to try to minimize their impact via the optimum use of whatever means are available. To achieve this goal, we need specific information such as: Which virus strain will appear next? When will the outbreak begin in our country? How long will it last? How dangerous will the 
epidemic be in terms of morbidity and mortality? In addition to its obvious epidemiological importance, such information may also have many practical uses.

\section{ACTIVE SURVEILLANCE FOR ACTION}

There is a large difference between information collected for 'academic surveillance' or a posteriori description of an epidemic's evolution, and an active system of surveillance for action. Our experience, after several winters, has shown that early information in the field of surveillance is much more useful than was initially thought.

Vaccine manufacturers, in conjunction with national health authorities, need long lead times to ensure that the most appropriate virus strains are included in the vaccine formulations, and to adapt these strains to optimum growth conditions for full scale production. Which virus strains are to be included in the vaccine for the next season in the northern hemisphere is decided in February each year at an international level by a World Health Organization (wHO) committee of experts, according to the information available on the prevailing strains and their characteristics. Thus, the availability of as many isolates of fresh virus strains as possible from different regions of the world is essential for a correct appreciation of the situation, and it is surveillance programs which can, and must, provide this service.

The above also applies, perhaps even more importantly, to the use of specific antiviral agents which depend upon the knowledge of the type of virus responsible for the outbreak, since some of these agents are only active against influenza A type viruses.

The vaccine should be administered before the start of the outbreak; information campaigns are started in October each year to remind people belonging to the high risk groups that it is time to receive the vaccine. These campaigns last until December but sometimes, as in November 1989 for instance, an outbreak begins early, even before the end of the vaccination season. It then becomes important to tell those people who forgot, or hesitated, to receive the vaccine, and that the epidemic will be at its peak in four weeks, so they are still able to be vaccinated in time.

Practitioners will appreciate advance notice of even a few weeks about when the epidemic will start. This helps them to plan their activities and it provides them with a very useful diagnostic tool. Knowing immediately which virus is circulating in their region gives them clues to assist in the clinical diagnosis of an acute respiratory infection.

Drug manufacturers, even if they are not directly concerned with antivirals, want to know when they will suddenly need increased supplies to deliver to the pharmacists because of an upsurge in consumption of everything from antipyretics to antibiotics, vitamins and cough preparations. A recent example of what can occur if this type of information is not available is exemplified by the case of one antibiotic manufacturer who ran out of stock two days after the beginning of an outbreak. This situation developed because of a combination of factors, including low storage reserves and a shortage of staff because of the epidemic. This resulted in a loss of market share to the company's competitors.

Forewarning of outbreaks, even in the short term, is also of interest to other professions which cannot stop or slow down their activities and have to consider an eventual replacement of missing staff due to an epidemic. It is also possible after analysis of these data to obtain a better evaluation of the economic losses caused by the epidemic. This can be calculated in terms of expenses directly due to the disease (excess medical expenses, drugs, etc) and in terms of losses due to a decrease in working capacity. Surveillance can also help to evaluate the efficacy of control measures.

Finally, information for the general public is of great importance as it controls the circulation of false or exaggerated rumours. Using the provision of information on the causative agent in circulation as a vehicle to explain what to do in case of a severe influenza outbreak is of practical use.

\section{THE ROLE OF PRACTITIONERS IN EPIDEMIOLOGICAL SURVEILLANCE}

Our conceptions of influenza epidemiology are biased as a result of dependence on data obtained primarily from the study of the most severe case reports, usually those seen in hospitals. There are generally not enough data on influenza as it presents in the general population. Observing influenza from the point of view of the practitioner in the clinic is not a common attitude. The study of hospitalized cases is of course very important and provides information on the virus itself, the strains in circulation and the main virulent variants. But the actual circulation of the agent in the bioenvironment is not fully characterized by this approach. 'Real' cases of influenza are those seen at home by first line practitioners. At the beginning of an outbreak the clinical picture may be mild and not quite characteristic. However, the type of virus in circulation is an indication of the actual danger of an epidemic occurring.

Surveillance has to be established at the level of general practice; the primary cases, not necessarily the most severe, need to be recorded. These include cases in families, in patients of all ages in the general population, in schools, in military recruits, in nursing homes, and with special attention given to children since they are known to be the first to be attacked by a new epidemic agent and they play an important role in later virus dissemination.

Including practitioners in a network where they have the responsibility of taking samples from patients and getting a rapid identification of the causative agents 
brings some surprises, first of all for the physicians themselves. Some of them can be very surprised at first to receive a negative result for a case with a typical influenza-like syndrome, for which they then have to look for another explanation. Or, in contrast, they will be surprised to receive a result of isolation of a true influenza A or B type virus from a sample taken from a child with a suspected case of common rhinitis without fever or with an undefined febrile condition. Some sentinels would now not miss an opportunity to sample, and have made a reputation for themselves as 'flu experts in their practices because, after having seen several outbreaks, they are able to recognize much more efficiently than before a true epidemic influenza picture. Of course this is much easier during the peak of an outbreak, when cases begin to be similar to each other, than at the start when the clinical picture is not yet stabilized. For the epidemiologist, it is interesting to observe the start of an epidemic, with a phase of (almost) silent circulation of the agent in the population and the crescendo of the phenomenon with cases spreading into new regions, with peaks and ebbs. The interval between the first isolation, the confirmation of an epidemic burst and the peak is of the utmost interest since it allows short term predictions (four to six weeks in advance) of the development and the possible extension of the outbreak. It is also interesting to observe the failure of an outbreak as was seen with the influenza type B strain imported from California to Paris in January, 1989.

\section{REGIONAL GROUPS FOR INFLUENZA OBSERVATION}

Since a network of physicians was to be established in France for the collection of data, it was a simple matter to extend the function of the network to include the collection of samples. The advantage of incorporating this additional function is that it provides the physicians with a good diagnostic control system, which thereby improves the accuracy of their diagnostic capabilities, and therefore increases their motivation to cooperate in the network.

Using these principles, the Groupes régionaux d'observation de la Grippe (GROG) system was evolved. GROGs are early warning networks for the detection and surveillance of influenza outbreaks. This system was designed in 1984 to complement the wHO influenza surveillance system and has been operational since that time, collecting specific and nonspecific data, transforming this information week after week into epidemiological indices and graphs. The first GROG was trialled in the ile-de-France region (Paris and surrounding area) during a three-year period, and after evaluation, the program was extended to all regions of France. The GROG system uses more than 20 specific and nonspecific indicators, and the computerized data are collected each Monday. French national GROG data are collected by nearly 1000 sentinels (virologists, gen- eral practitioners, pediatricians, pharmacists, hospitals, social security centres, army medical services, etc) under the responsibility of wHO Influenza National Reference Centres (Institut Pasteur, Paris and Laboratoire National de la Santé, Lyon). GROGs are coordinated by two general practitioners.

The results for the past few winters show that systems such as GROG allow the early detection of influenza virus activity and provide a short term warning with regard to the development of an outbreak in a given geographically homogeneous community. The last epidemics were forecast and their peaks predicted four to five weeks beforehand. This does not mean that it is safe to wait for this alert before implementing vaccine use, since during this four- to five-week period when the epidemic is building up, millions of patients are already suffering from influenza and thousands may die.

\section{PRACTICAL CONDITIONS OF SURVEILLANCE}

Data collection by a representative sample of practitioners is a very simple way to obtain information, but the data are exclusively of a clinical and practically a micro-epidemiological nature. However, other types of useful information are also easily available from other sources, which include: absenteeism reports; the consumption of drugs; and the level of general practitioner activity, especially of those involved in home care.

The collection of these 'nonspecific data' can be validated by comparison with other types of data such as clinical and virus isolation records. This is aided by the availability of rapid diagnostic methods which have become an important tool in this field. For example, it is now possible to obtain, at relatively low cost, the identification of the main cause of the viral respiratory infection in question within $4 \mathrm{~h}$ after the sample has been taken from the patient.

In order to develop this system fully, it is necessary to solve several logistical problems, such as the type of samples to be taken, the mode of shipment, the type of tests to apply and the mode of distribution of the information.

Samples: Kits for taking samples are provided to all sentinel physicians. These include: a swab; a vial containing transport medium; instructions for sampling; a simple patient history card to be completed; a mailing box; and a self-addressed envelope. This kit has been designed to make the operation as simple as possible for the physician. The material is then sent by messenger or by mail to the laboratory and, because of this phase, the effect of transport on the efficiency of isolation under our conditions, even for rather labile agents such as respiratory syncytial virus (RSV), was investigated and found to be negligible.

Sample treatment: Samples received at the laboratory are processed immediately according to a standard protocol. They are tested by a rapid diagnostic tech- 
nique using an enzyme-linked immunoadsorbent assay (ELISA) derived immunocapture method, and the answer is available within 1 to $4 \mathrm{~h}$. This early result is sent immediately to the physician. It is usually very specific, but less sensitive than culture, and false negative readings can be obtained. At the same time, the sample is inoculated into cell cultures (Madin-Darby canine kidney continuous cell line) and the culture is observed every second day for two weeks by the immunocapture test for the appearance of hemagglutinin. This protocol is equally efficient for A and B type viruses and could be adapted easily to other respiratory agents such as RSV, adeno- or parainfluenza viruses.

Distribution of information: The information concerning the isolates and the evolution of indices is dealt with rapidly every Monday and is sent on Tuesdays to the authorities, the participants in the network and to all other destinations where the information is considered useful. This includes dissemination in publications (daily and medical journals) to the medical profession and to the public. This type of information is very useful for vaccination programs since it is directed at people in the 'high risk' groups, and illustrates the benefit of vaccination and what the real problem is, and helps people to be aware of the potential danger influenza poses. Vaccination campaigns can make use of the results of previous experiences and may adapt the recommendations arising from these previous experiences to the current influenza situation.

\section{VACCINE POLICIES}

Since the late 1960s, highly concentrated and purified vaccines have been available and have proven to be effective in preventing influenza in at least $70 \%$ of recipients if vaccine composition matches the current epidemic strains. This has been documented by hundreds of publications and reports in many countries; a few reports of failures are easily explained by identified factors such as inappropriate vaccine composition, defects in the vaccine itself and factors related to the recipients and their inability to respond to immunizations. The generally adopted vaccination policies in western countries are to advise physicians to immunize their high risk patients before the beginning of winter each year and to inform the target groups through public information campaigns. These actions vary in different countries according to the organization of the medical system, the local modes of practice, the age and degree of concern of the population, and the priority and amount of financial support applied to the problem. In many countries, vaccine is paid for by the state insurance system or by a social security agency.

In addition, the role of physicians - family doctors for instance - is an important factor. It is imperative, for vaccination programs to succeed, that practitioners are themselves convinced of the usefulness of the vaccine. They will then be equipped to discuss the subject in an informed manner with their identified high risk patients and will therefore be able to recommend an appropriate course of action in an appropriate time frame.

The patients, of course, also play an important role. They learn quickly to recognize whether they belong to a high risk group and they will often take the initiative to ask their physician if, as a result of their condition, they are candidates for the vaccine.

This does not mean that sufficient effort is always made to enforce vaccination in all cases. The rates of vaccination have been found to be strikingly different in neighbouring countries of equivalent developmental status. In western countries, it is estimated that no more than 8 to $10 \%$ of high risk patients were vaccinated during the past winters, making "influenza ... a preventable disease not being prevented". However, in France, after 10 years of effort and the participation of state agencies, this rate has increased from $20 \%$ in 1970 to $70 \%$ in 1990 with a rate of $13 \%$ if the number of vaccine doses used is applied to the whole population.

One main reason for this excellent result is the financial contributions of the National Social Security System, which is saving money on disease expenses during the epidemics. The second important factor contributing to this success is the effort made to present information in a constant, clear and honest manner through professional channels and public media, on vaccines and on the development of epidemics in the country and in the world. 


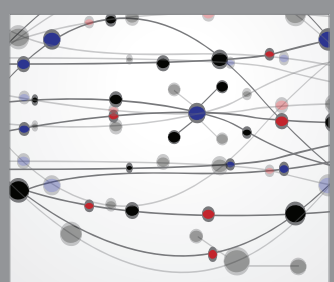

The Scientific World Journal
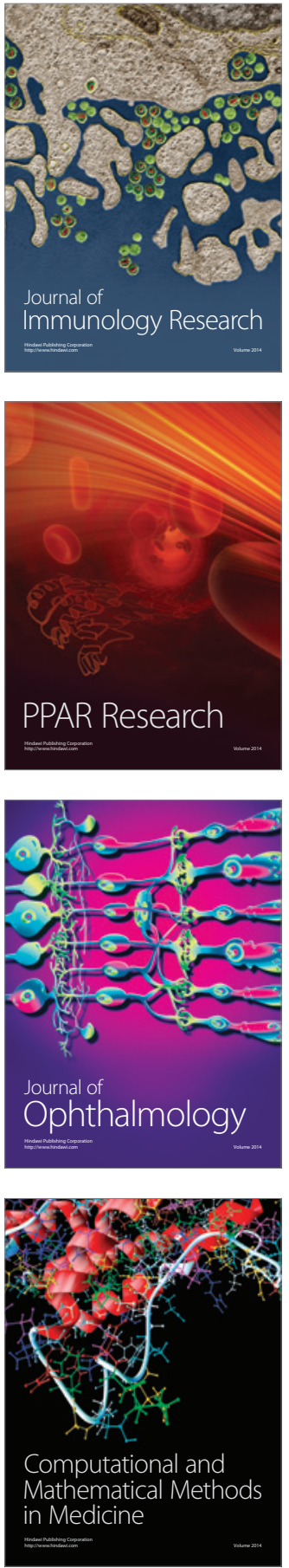

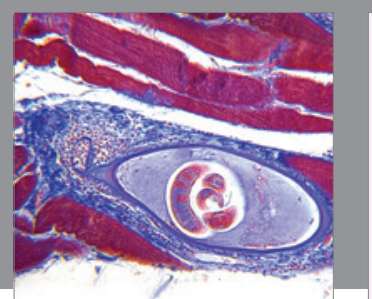

Gastroenterology Research and Practice

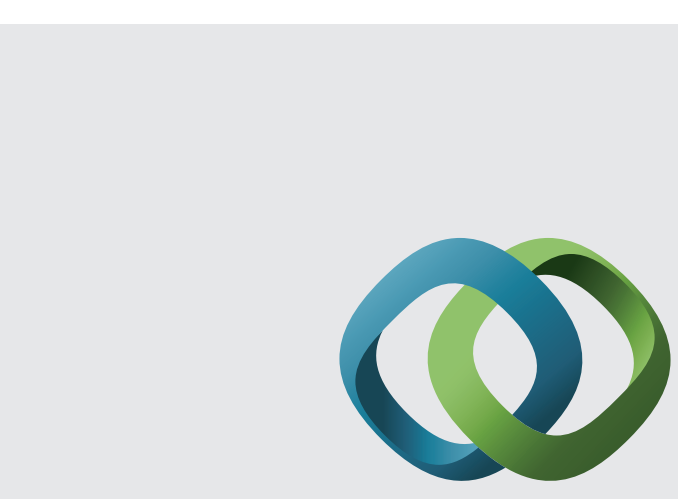

\section{Hindawi}

Submit your manuscripts at

http://www.hindawi.com
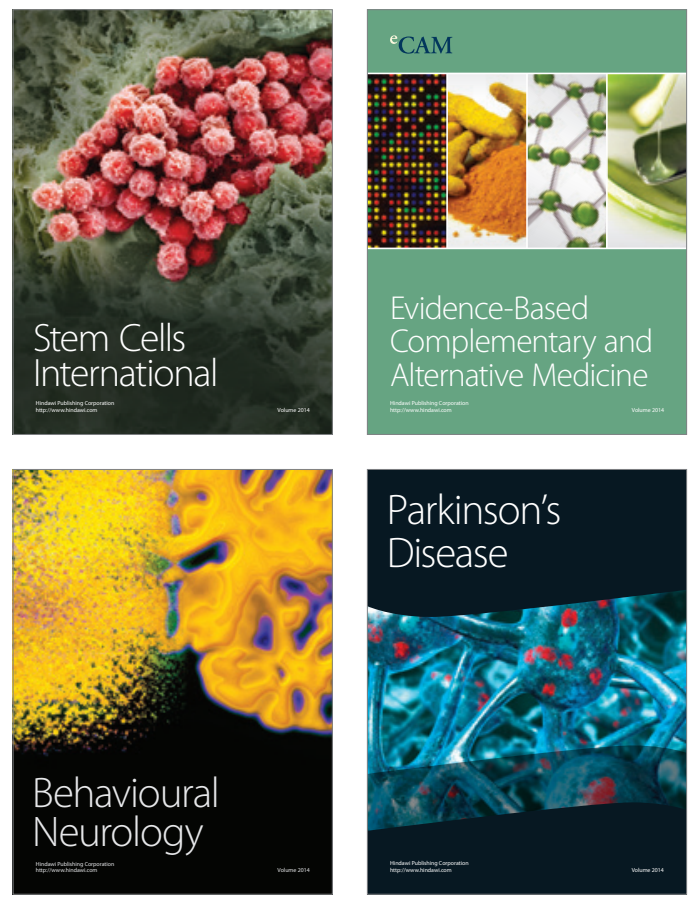
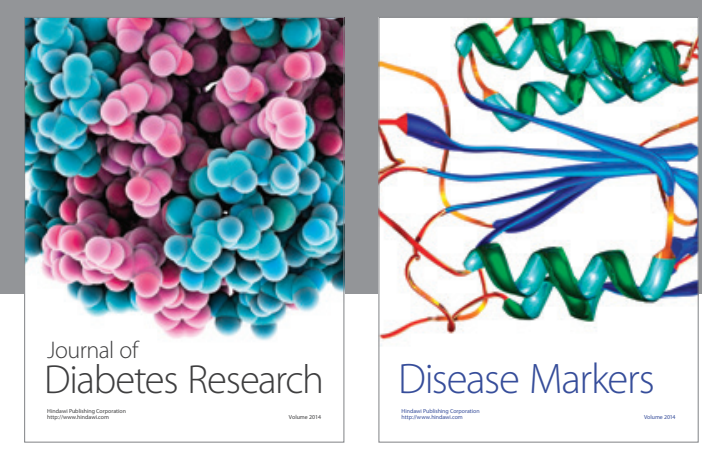

Disease Markers
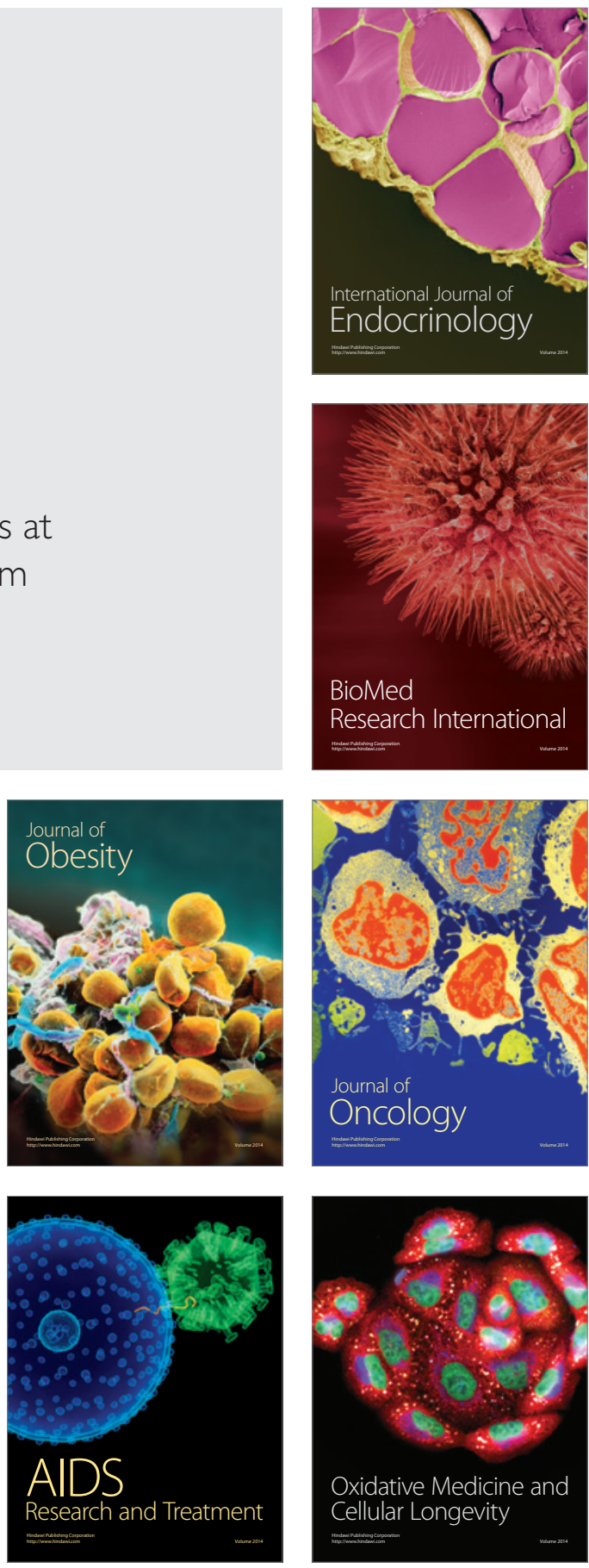